\title{
Therapie Sucht Krankheit
}

\section{Ralf Schrader}

Der nachfolgende Beitrag stellt nach Einschätzung der Redaktion ein eindrückliches Zeugnis eines individuellen Wegs dar. Mögliche problematische Aspekte und Folgen der Veröffentlichung wurden von der Redaktion sorgfältig erwogen und mit dem Autor besprochen. Er entschloss sich nach reiflicher Bedenkzeit dazu, den Text der Leserschaft in der nun vorliegenden Form zu unterbreiten ein freier Entscheid, den es nach unserer Auffassung zu respektieren gilt.

Die Redaktion

Ich bin Alkoholiker, so nennt man Leute, die, wenn sie anfangen Alkohol zu trinken, damit nicht wieder aufhören können. Eigentlich ganz einfach, man darf nicht anfangen, dann gibt es auch kein Problem mit dem Aufhören.

Seit ein paar Jahrzehnten gibt es hartnäckige Versuche, uns zu pathologisieren. Verständlich, Therapeuten kommen sonst nicht an das Geld der Krankenkassen, bei uns selbst ist irgendwann nicht mehr viel zu holen. Vor allem hat die wettbewerbs- und mittigorientierte Humanumgebung den dringenden Wunsch nach Abgrenzung. Da ist «krank» ein schönes Etikett, es disqualifiziert ausreichend, hat aber dennoch den Aspekt des Mitgefühls.

Vor 50 Jahren haben Alkoholiker kaum jemanden gestört, die Krankheitserklärung stammt erst aus 1976. In jedem Dorf gab es einen Dorftrottel und je nach Grösse eine Handvoll oder Dutzende Alkoholiker. Es war selbstverständlich, sie zu schützen, und Auseinandersetzungen waren nur intern zulässig. Die Trottel und Alkoholiker durften in weiten Grenzen machen, was sie wollten.

Unter uns haben wir die manchmal vorgeführt, aber Fremden haben wir das nicht gestattet. Das wussten alle Fremden, die haben es mit den ihren nicht anders gemacht. Psychologisch gesehen war die Welt damals komplett in Ordnung, man brauchte eine Zehnerpotenz weniger Psychotherapeuten und das Allheilmittel war

\section{Ich möchte nicht für krank gehalten werden.}

Retardiert, behindert kann alles sein, aber nicht krank.

Das kann nur sagen, wer kein richtiges Verständnis

\section{für den Krankheitsbegriff hat}

Korrespondenz:

Dr. med. Ralf Schrader

Lindenstrasse 16

CH-6005 Luzern

ralf.schrader@gmx.ch
Akzeptanz. Menschen müssen nicht perfekt sein, die dürfen sogar sehr daneben sein. Man muss es ihnen gestatten, mehr braucht es nicht. Die perfekt sein wollen, gehen in Psychotherapie. Nur brauchen wir kaum perfekte Menschen. Perfekt bedeutet heute im wesentlichen berechenbar sein, berechenbar für die Politik, die Wirtschaft und für die Werbung.

Opferrollen entstehen vor allem durch Einreden, das hat damals keiner gemacht. Es gab vor 50 Jahren kein Borderline-Syndrom, kein
ADHS, in islamischen Ländern gibt es das auch heute nicht.

Warum produzieren wir denn so viele Danebenliegende? Weil etwas gefordert wird, was nur selten einzuhalten ist. Ich will schon deshalb nicht normal werden, weil ich diese ganze Art nicht mag. An mir ist nichts Pathologisches, ich bin gesund und ziemlich robust. Aber ich will mich mit nichts ausser mir selbst arrangieren. Ich will nicht da hinkommen, wo derzeit das Menschenbild ist, das aus dem Businessplan eines Zuchtgestüts entlehnt scheint. Arbeiten und Geben mache ich gern, und wenn mich jemand angreift, dann ziehe ich mich zurück. Selbst greife ich nicht an.

Man muss Menschen auch in Frieden leben und sterben lassen und nicht ständig an ihnen herum manipulieren. Muss man ihnen aber beizeiten sagen und sie nicht in dem Glauben lassen, dass es immer eine Intervention gibt.

Ich bin nicht krank. In der frühen Entwicklung ist bei mir etwas schief gelaufen, Freud würde das als Nichtentstehen des Urvertrauens diagnostizieren, ich bin mir sicher, der hatte das auch. Ich bin also behindert, für die Krankenkasse sollte das reichen.

Ansonsten nützt es mir wenig, das zu wissen. Es ist nämlich nicht rückgängig $\mathrm{zu}$ machen. Eigentlich sollte man glauben, dass Verschaltungen von Neuronen immer wieder herstellbar sein sollten. Ist aber nicht der Fall. Ich habe mein ganzes Leben auf den Alkoholismus, auf die zugrundeliegende Behinderung abgestimmt. Ich bin affektiv retardiert, dafür habe ich Sensibilität und kompensatorisch intellektuelle Mechanismen entwickelt. Ich kann fast jede Rolle spielen, die von mir erwartet wird, immer vorausgesetzt, dass ich wenigstens drei Tage nicht getrunken habe. Trauer, Aggression oder Sexualität zu spielen fällt schwer, aber meistens bemerkt das keiner. Wer beobachtet, bemerkt es natürlich.

Das macht aber kaum einer, weil jeder vorrangig mit sich, besser mit seiner Aussenhaut beschäftigt ist. Ich habe längst aufgegeben, mich mit meiner Aussenhaut zu beschäftigen. Deshalb kann ich beobachten und da ich selber nur imitiere, erkenne ich andere Imitatoren schon nach Minuten. Es fällt mir, wenn ich nicht trinke, unheimlich schwer, meine Umwelt zu ertragen. Ich habe nichts dagegen, wenn einer mir etwas vorspielt, in der Gewissheit zu spielen. Dann können wir uns famos ergänzen. Aber es ödet mich 
an, wenn jemand in dem Glauben spielt, echt zu sein. Bisher habe ich nur wenige Menschen kennengelernt, die mir echt seiend erscheinen. Das wirkt dann manchmal richtig albern, weil «echt» nie «kontrolliert» sein kann. Ich bin fast nur kontrolliert und wenn ich echt bin, dann bin ich peinlich. Mit echten Menschen kann ich gut interagieren.

Ich möchte nicht für krank gehalten werden. Retardiert, behindert kann alles sein, aber nicht krank. Das kann nur sagen, wer kein richtiges Verständnis für den Krankheitsbegriff hat. Wenn ich noch für längere Zeit trocken bin, schreibe ich etwas dazu.

Allerdings, oder gerade deshalb: «Es gibt Patienten, die geradezu triumphierend ihre Diagnosenliste runterleiern.» (mündlich vorgetragenes Zitat) Kranksein ist auch ein schützendes Attribut und wer es hat und es haben will, soll es behalten.

Es wird viel Geld eingesetzt, um uns sozial-, besser gesellschaftskompatibel zu halten. Das wäre auch so zu lösen: Ich bekomme einen amtlichen Zettel, auf dem steht, dass ich Alkoholiker bin, und den bringe ich zum Sozialamt. Dieses zahlt dann meine Miete und schickt den Rest des Existenzminimums an den Discounter der Wahl, der mir jeden Morgen einen gefüllten Einkaufskorb in den Flur stellt. Es ist peinlich, mit Mundgeruch und äusserlich verlottert, einkaufen gehen zu müssen.

\section{Ich bin aber auch der lebende Beweis, dass diese} ganzen Suchttheorien nicht stimmen. Ich bin alkoholabhängig, aber nicht generell süchtig

Jedes halbe Jahr kommt jemand gucken, ob ich nicht aus Versehen trocken und damit arbeitsfähig geworden bin. Das kann dann nämlich passieren, von ganz allein. Therapie ist teurer.

Ich schliesse meine Tür nicht mehr zu, sage allen Nachbarn, was mit mir ist, und sage ihnen auch, dass sie jederzeit unangemeldet hereinkommen dürfen. Aushalten müssen sie den Anblick, nicht ich. Dann ist kein Risiko für meine Umgebung, unkontrolliertes Verhalten haben trainierte Alkoholiker sowieso nicht.

Lange müsste das Sozialamt auch nicht zahlen, entweder saufe ich mich schnell zu Tode oder ich bekomme selbstorganisiert den Absprung. Nur dann fangen die Probleme richtig an. Wer will mit einem wie mir zu tun haben? Ich habe nichts von den Eigenschaften, die gefragt sind.
Ich bin nicht teamfähig, nicht konsensfähig, nicht partnerschaftsfähig. Das war ich schon als Kind nicht. Deshalb ist es nicht unbedingt ein Ausdruck von Humanität, mich vor dem Abstieg oder dem Tod zu bewahren.

Ich bin aber auch der lebende Beweis, dass diese ganzen Suchttheorien nicht stimmen. Ich bin alkoholabhängig, aber nicht generell süchtig. Als Arzt kann ich mir alles beschaffen, aber ich habe es, von dümmlichen Versuchen abgesehen, nie gemacht. Wenn mich morgens um 2 Uhr der Entzug ereilt, ertrage ich den notgedrungen, bis der nächste Laden öffnet. Ich habe alles im Haus, nach Todeserfahrungen möchte ich nicht qualvoll sterben, ich merke, wann es soweit ist. Aber ich nehme nichts, um mich zu sedieren oder um die eine Droge durch die andere zu ersetzen. Alkohol liebe ich, andere Suchtmittel sind mir gleichgültig.

Wenn man so ist, muss man ein Verhältnis dazu bekommen. Diese Krankheitsgeschichte ist dafür überhaupt nicht hilfreich und der Freud'sche Kram auch nicht. Verteilungen haben Ränder, und da muss auch jemand sein. Wenn ich es nicht wäre, wäre es ein anderer. Die Gesellschaft muss lernen, dass sie alles ist, nicht nur der gute Mittelteil. Wie immer etwas konfiguriert ist, rechts und links gibt es Abweichungen. Eine Gesellschaft ist genau so stark, wie sie Abweichungen tolerieren kann. Ich bin eine Abweichung, und die muss man vor allem ertragen.

Mit dem Alkoholismus werde ich zu Rande kommen, wir haben Waffenstillstand vereinbart. Alles andere bleibt. Man kann versuchen mich zu therapieren, aber eben mich, nicht den Alkoholismus und nicht die zugrundeliegende Behinderung. Ich habe gerade eine Therapie hinter mir, die hat mich nachhaltig verändert. Aber ob das besser als vorher ist, muss sich noch herausstellen. Ich bleibe am Rand, nur ist es jetzt vielleicht ein anderer Rand. Ich bin kein Fall für die Medizin, auch nicht für die Psychologie. Wenn man mich aus meinen Wurzeln reisst, kann das meine Oberfläche polieren, die inneren Spannungen werden stärker. Insbesondere zerreisst es mich, wenn ich mich anpassen muss.

Am besten ist es tatsächlich, uns einfach in Ruhe zu lassen, es sei denn, wir leiden und suchen Hilfe gegen Leid. Wenn man mich liebevoll in Ruhe lässt, steigt mein Scham- und Verantwortungsgefühl und ich versuche zurückzugeben und mich zurückzunehmen. Korrigieren kann man nichts mehr, das ist einfach mal gelaufen. Das Leben in einen halbwegs sinnvollen Kanal selbstorganisierend gestalten kann ich noch machen. Das ist aber kein primär therapeutischer, sondern ein sozialer Ansatz. 